\title{
Prevalence of and risk factors for multidrug-resistant tuberculosis in Iran and its neighboring countries: systematic review and meta-analysis
}

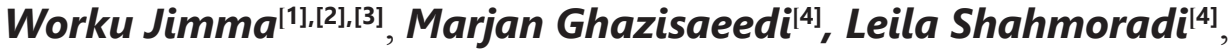 \\ Ahmed Abdulahi Abdurahman ${ }^{[3],[5],}$, Sharareh Rostam Niakan Kalhori ${ }^{[4]}$, Mahshid Nasehi[1],[8], \\ Sharifi Yazdi ${ }^{[6]}$ and Reza Safdari[4]
}

\begin{abstract}
[1]. Department of Health Information Management, School of Allied Medical Sciences, Tehran University of Medical Sciences, International Campus, Tehran, Iran. [2] Department of Information Science, College of Natural Sciences, Jimma University, Ethiopia. [3] Students' Scientific Research Center, Tehran University of Medical Sciences, Tehran, Iran. [4]. Department of Health Information Management, School of Allied Medical Sciences, Tehran University of Medical Sciences, Tehran, Iran. [5]. Department of Community Nutrition, School of Nutritional Sciences and Dietetics, Tehran University of Medical Sciences, International Campus, Tehran, Iran. [6]. Department of Medical Laboratory Sciences, School of Allied Medical Sciences, Tehran University of Medical Sciences, Tehran, Iran.
\end{abstract}

[7]. Center for Communicable Diseases Control, Ministry of Health and Medical Education, Tehran, Iran.

[8] Department of Epidemiology and Biostatistics, School of Public Health, Iran University of Medical Sciences, Tehran, Iran.

\begin{abstract}
Tuberculosis, in particular drug-resistant tuberculosis, is of global concern due to the high mortality and morbidity associated with it annually. The aim of this study was to determine the prevalence of and the risk factors for multidrug-resistant tuberculosis in Iran and its neighboring countries. Four databases (PubMed, BioMed Central, EMBASE, and Web of Science) were searched using key terms. Nineteen eligible articles were identified, of which 12 and seven were used for quantitative and qualitative analysis, respectively. The overall pooled estimate of the prevalence of multidrug-resistant tuberculosis, including both new and previously treated tuberculosis cases, in Iran, Iraq, Turkey and Pakistan was 16\% (95\% confidence interval [CI] 11-20). The patients with a previous tuberculosis treatment history (odds ratio $[\mathrm{OR}]=6.45 ; 95 \%$ CI 5.12-7.79), those aged $<45$ years (OR $=1.57 ; 95 \% \mathrm{CI} 1.12-2.03)$, and those who were males $(\mathrm{OR}=1.83 ; 95 \% \mathrm{CI} 1.19-2.48)$ had an increased pool risk of developing multidrug-resistant tuberculosis. The forest plot revealed that the pooled odds for the development of multidrug- resistant tuberculosis were 2.01 (95\% CI 1.65-2.36). Poor adherence to treatment was one of the predictors of unsuccessful treatment outcomes. Multidrug-resistant tuberculosis is a great concern for public health programs in many countries globally, including those included in this review. The risk factors for the development of multidrug-resistant tuberculosis, specifically a previous tuberculosis treatment history, should be targeted through the implementation of specialized interventions.
\end{abstract}

Keywords: MDR-TB. Prevalence. Risk factors. Treatment. Patient care.

\section{INTRODUCTION}

Tuberculosis (TB) remains to be one of the deadliest infectious diseases in the world; in 2015, there were 10.4 million new cases and 1.8 million deaths due to TB and TB-HIV co-infection worldwide ${ }^{1}$. The development of drug resistance during the course of TB treatment, leading to multidrug-resistant tuberculosis (MDR-TB), poses even greater risks for long-term survival of patients. MDR-TB is a major health hazard and is associated with sub-optimal rates of treatment success and concurrent high rates of mortality ${ }^{2,3}$.

It was reported that between 2000 and $2004,20 \%$ and $2 \%$ of TB cases were resistant to standard first-line and second-line TB drugs, respectively ${ }^{4}$. Resistance develops either when a person

Corresponding author: Dr. Marjan Ghazisaeedi.

e-mail: ghazi.saeedi@yahoo.com

Received 4 April 2017

Accepted 18 May 2017 is infected with a resistant strain or when improper treatment leads to drug selection of resistant strains. Drug resistance has become a serious public health problem in a number of countries and an obstacle to effective TB control. According to the World Health Organization (WHO), an estimated 3.5\% and $20.5 \%$ of new and previously treated TB cases were estimated to have MDR-TB; in 2015 it was estimated that 480,000 people developed MDR-TB ${ }^{1}$. The WHO also reported that there were also 100,000 people with rifampicin-resistant TB (patients with resistance to at least rifampicin) who were newly eligible for MDR-TB treatment in 2015.

MDR-TB is multi-factorial and fueled by improper treatment of patients, poor management of supply and quality of drugs, and airborne transmission of bacteria in public places. Case management becomes difficult and is compounded by catastrophic economic and social costs that patients confront while seeking assistance and while on treatment. Effective management of MDR-TB requires input for activities related 
to prevention, case detection, treatment and care, surveillance, drug management, and monitoring as well as evaluation of the program's performance ${ }^{1}$.

Iran shares geographic borders with three countries where TB is endemic (Pakistan, Iraq, and Afghanistan) ${ }^{5}$, thus TB control remains one of the main public health concerns and a priority among public health policy makers. Though the reported TB incidence rate for Iran has decreased from 36/100,000 in 1990 to $17 / 100,000$ in $2010^{5}$, it is estimated that the number of MDR-TB cases annually in Iran is 1,305; prevalence rate is approximately $5 \%$ and $48.2 \%$ among new and retreatment TB cases $^{2}$. Another study revealed that the reported and estimated number of TB cases in Iran was 17 and 52 per 100,000 persons, respectively; whereas in Afghanistan, there were 44 and 314 reported and estimated cases per 100,000 persons, respectively ${ }^{6}$.

Large numbers of immigrants from Afghanistan have come to Iran and due to the high prevalence of TB in Afghanistan, it is believed that there are considerable numbers of cases with active, latent, and multiple drug resistant TB infections among those of different nationalities in Iran ${ }^{6,7}$. Various studies have indicated the trend in TB transmission among Iranian and Afghan nationals based on molecular tests ${ }^{7,8}$. The movement of people in the region is common as people seek job opportunities, religious pilgrimage, or trading; this migration is likely to have impacted the TB and MDR-TB prevalence in these endemic countries $^{5}$. Thus, conducting a systematic review and metaanalysis of countries in the Middle East, endemic for MDRTB, will provide comprehensive information about MDR-TB which will be useful for stakeholders, such as policy makers, health professionals' and researchers, in the development and implementation of appropriate interventions. The aim of this systematic review and meta-analysis was to determine the prevalence of MDR-TB and the risk factors associated with the transmission of and development of MDR-TB in Iran and its neighboring countries, including Turkey, Iraq, Pakistan, Afghanistan, Armenia, Azerbaijan, and Turkmenistan. Additionally, we wished to ascertain information as to the management of MDR-TB patients, including the specific treatment and care provided to patients, based on the data and information obtained from cross-sectional or cohort studies conducted in these countries.

\section{METHODS}

\section{Design}

This was a systematic review and meta-analysis of MDRTB patients.

\section{Search strategy}

For the present study, the guidelines for Preferred Reporting Items for Systematic Reviews and Meta-Analyses (PRISMA) were followed ${ }^{9}$. The databases used for this study included PubMed, BioMed Central, EMBASE [(Excerpta Medica dataBASE) is a biomedical and pharmacological database)], and the Web of Science. Only publications in English language were considered and no restrictions on publication dates were imposed. The key terms used to search relevant articles in these databases were multidrug-resistant tuberculosis, prevalence, risk factors, and patient management for all the countries considered for this study. Accordingly, the databases were searched using each key term by appending the countries name. Data and/or information were extracted from the selected articles to answer the various research questions. All these methods were performed by two of the authors (Jimma W and Abdurahman AA) independently. When discrepancies arose whether to include or exclude studies for analysis, a consensus was reached by obtaining inputs from the third author as a tiebreaker. The steps employed to select eligible articles for this systematic review and meta-analysis is depicted in Figure 1.

An example of the search details for PubMed is as follows:

(Tuberculosis, Multidrug-Resistan [Mesh]) AND (prevalence [tiab]) OR occurrence* [tiab]) OR incidence* [tiab])) OR (((risk factors [tiab]) OR determinant* [tiab]) OR cause* [tiab]))) AND ((((patient management [tiab]) OR treatment [tiab]) OR care [tiab]) OR drug administration [tiab])) AND (c(((c((Iran [tiab]) OR Turkey [tiab]) OR Iraq [tiab]) OR Pakistan [tiab]) OR Afghanistan [tiab]) OR Armenia [tiab]) OR Azerbaijan [tiab]) OR Turkmenistan [tiab])

\section{Inclusion and exclusion criteria}

Original research articles on MDR-TB from the target countries and those published in English language were included, whereas articles in other languages, publications on extensively or total drug resistant tuberculosis, systematic review and/or meta-analysis articles, and conference abstracts were excluded.

\section{Study quality and risk of bias assessment}

For this review, the Newcastle-Ottawa quality assessment scale was employed to assess the risk of bias of the included studies; two of the authors (Jimma W and Abdurahman AA) independently assessed the studies and those with a low quality score and were excluded from the sensitivity analyses. Weight was assigned based on eight pre-determined criteria; studies that obtained a score of 8-9 were considered as high quality studies with less estimated risk of bias and were therefore used in the sensitivity analyses.

\section{Data and information extraction and synthesis}

The data and information extracted from all the eligible studies included: authors, study location (country), year of publication, time of data collection, study design, sample size, prevalence of MDR-TB, risk factors associated with MDR-TB, demographic characteristics of the study participants (such as age, sex), the minimum and maximum duration of the treatment, and treatment outcomes.

The variations in MDR-TB patients' management such as, the drugs administered, drugs withdrawn, or regimen changes, as well as other pertinent information was synthesized narratively and presented.

\section{Statistical analysis}

Heterogeneity $\left(\mathrm{I}^{2}\right)$ between the studies was checked by testing the null hypothesis, the presence of homogeneity in the prevalence reported in the studies. $I^{2}$ describes the total variation 


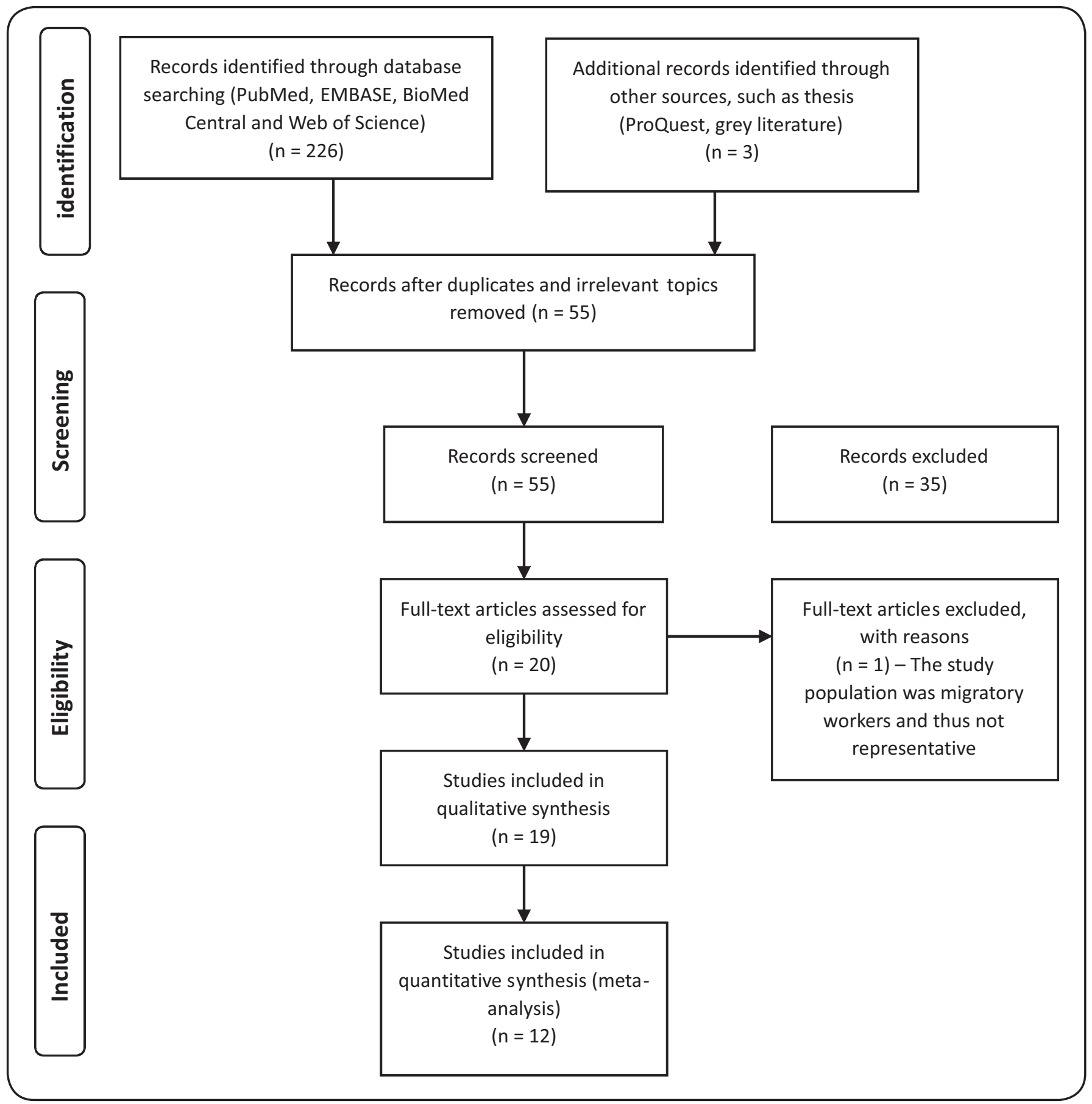

FIGURE 1 - PRISMA flow diagram for the systematic review and meta-analysis. EMBASE: biomedical and pharmacological database; PRISMA: Preferred Reporting Items for Systematic Reviews and Meta-Analyses.

across studies and is used to identify violation in the assumption of homogeneity where $\mathrm{I}^{2}>60 \%{ }^{10}$.

Statistical analysis was carried out using the statistical software package Stata version 13 (Stata corporation, college station, Texas, USA). The effect size of the meta-analysis was conducted for the prevalence of MDR-TB in Iran and its neighboring countries. The risk factors for the development of MDR-TB were examined for the studies that met the meta- analysis eligibility criteria, i.e., when a risk factor was reported by at least two studies (indicated by the odds ratios [ORs] and $95 \%$ confidential intervals [CIs] reported in each study). A random effects model was used to obtain the pooled effect size of the individual risk factors taking into account both the sampling error and the heterogeneity between studies using the generic inverse variance method (i.e., the metan command in Stata). Effect size was pooled from all eligible studies using 
the DerSimonian-Laird Random effects model (REM) for the meta-analysis ${ }^{11}$. This model is preferred because it is based on the assumption that a distribution of effects exists, resulting in heterogeneity among study results ${ }^{12}$. The results were presented in a table as forest plots; additionally prevalence rates, ORs, and 95\% CIs were obtained for each study inserted into the model and for the overall estimate.

To determine the extent of publication bias, funnel plots were plotted and observed for asymmetry. Moreover, Egger's test was computed using the metabias command in Stata ${ }^{13}$. Duval \& Tweedie trim and fill methods were used to adjust the analysis for the effects of publication bias ${ }^{12}$. The effect of the deviant studies was examined via sensitivity analyses for the studies classified as high quality, after the exclusion of studies with low scores, based on the aforementioned quality scoring system.

\section{Ethicals considerations}

The protocol for this systematic review and meta-analysis was registered on the PROSPERO International Prospective register of systematic reviews, managed by the University of York. It was assigned the number PROSPERO 2016, CRD42016038392, and can be accessed on the following link: http://www.crd.york.ac.uk/PROSPERO/display_record. asp?ID=CRD42016038392. Informed consent was not required for this study as it was a review of already published data.

\section{RESULTS}

\section{Literature search and selection of eligible articles}

The search for articles using Medical Subject Headings $(\mathrm{MeSH})$ and key terms returned a total of 229 research articles of which 55 full text articles were retained after evaluation of the titles and abstracts. After reading the full text, 19 articles ${ }^{6,14-31}$ were considered eligible for this systematic review and metaanalysis, of which 12 articles were used for quantitative analyses of both prevalence of and risk factors for MDR-TB (Figure 1). Five studies reported both the prevalence of and risk factors for MDR-TB ${ }^{16,21,25-26,31}$. Our search strategy yielded eligible studies from the following countries: Iran, Iraq, Turkey, Pakistan, and Armenia. There was no study available or available but not eligible according to the inclusion/exclusion criteria from the remaining three neighboring countries (Afghanistan, Azerbaijan, and Turkmenistan).

The studies included were a mixture of prospective or retrospective cross-sectional or cohort studies, conducted in laboratory or hospital based settings 1992 to 2013. The number of MDR-TB patients included in the studies ranged from 172,367. In total, there were $776 \mathrm{MDR}-\mathrm{TB}$ cases identified among the 6,811 cases included in the analysis of prevalence, whereas there were 5,439 cases included in the analysis of risk factors for MDR-TB. The ages of the included patients ranged from 2-76 years.

\section{Care for MDR-TB patients}

The majority of the studies reported that large quantities of drugs were being used to treat MDR-TB patients, which were accompanied by numerous adverse effects. Moreover, MDR-TB treatment duration is extremely long, spanning between 20-24 months. Ahmed et al. ${ }^{14}$ reported that in Pakistan MDR-TB patients received a median of six drugs during the intensive phase. In Iran, MDR-TB treatment was initially initiated using a mean of 5.23 drugs $^{6}$ while the mean number of drugs used throughout MDR-TB treatment was $5.7^{28}$. In the study by Ahmed et al. ${ }^{14}$, the median duration of treatment for patients who were cured was 24 months (interquartile range [IQR] 2034). Mirsaeidi et $a l^{6}$ also reported that the average MDR-TB treatment duration was 27.9 months (IQR 12-96).

The adverse effects associated with MDR-TB treatment are barriers leading to lack of adherence to treatment. Bastard et $a l .{ }^{15}$ reported that poor adherence to treatment due to intolerance of the adverse effects was one of the predictors of unsuccessful treatment outcomes. Moreover, Sanchez-Padilla et al.$^{24}$ reported that the main reason for discontinuing treatment in all the patients who were interviewed was difficulty in dealing with side effects. Some of the studies reported the withdrawal of one or more drugs or modifications to treatment regimens as a result of adverse effects ${ }^{14,28}$. Table 1 presents the care for MDR-TB patients and the major findings of each study (Table 1)

\section{Prevalence of MDR-TB in Iran and its neighboring countries}

Ten studies were included for the meta-analysis of MDRTB prevalence ${ }^{16,18,20-22,25-27,29,31}$. The overall pooled estimate of MDR-TB prevalence in Iran, Iraq, Turkey, and Pakistan, was $16 \%$ (95\% CI 11-20) (Figure 2). The $\mathrm{I}^{2}$, test showed that there was statistically significant heterogeneity among the studies for the respective countries ( $\mathrm{p}<0.001)$; $\mathrm{I}^{2}$ was $97.93 \%, 98.97 \%$, $96.64 \%$, and $98.32 \%$ for Pakistan, Iraq, Iran, and Turkey respectively. The overall $\mathrm{I}^{2}$ of the studies in these countries was $97.79 \%(\mathrm{p}<0.001)$.

The pooled estimates of MDR-TB prevalence for the studies included for each country were: $5 \%$ (95\% CI 0-10) in Pakistan; $12 \%(95 \%$ CI $8-17)$ in Iraq; $27 \%$ [95\% CI 16-39] in Iran; $9 \%$ (95\% CI 7-12) in Turkey.

The funnel plot showed that there is bias in the studies as depicted in Figure 3. Thus, the egger test was performed which found that the publication bias was statistically significant ( $p$ $=0.03$ ).

\section{Sensitivity analysis}

Sensitivity analysis was performed after removing data from the studies that obtained low quality scores from the metaanalytic model. Seven studies with high quality scores were included. The prevalence of MDR-TB in Iran, Pakistan, and Turkey was $16 \%$ (95\% CI 14-17), 8\% (95\% CI 7-9), and 7\% (95\% CI 5-11), respectively (Figure 4). The $\mathrm{I}^{2}$ of the sensitivity analysis was $96.18 \%$ and it remained significant $(p<0.001)$, indicating that the quality score did not significantly impact the overall pooled prevalence of MDR-TB.

\section{Risk factor analysis}

The patients with a previous $\mathrm{TB}$ treatment history $(\mathrm{OR}=6.45$; $95 \%$ CI 5.12-7.79), those aged $<45$ years $(\mathrm{OR}=1.57 ; 95 \% \mathrm{CI}$ $1.12-2.03)$, and those who were males; $(\mathrm{OR}=1.83 ; 95 \% \mathrm{CI}$ 
TABLE 1

Studies on multidrug resistant tuberculosis patients' care and their main findings.

\begin{tabular}{|c|c|c|c|c|c|}
\hline Author & $\begin{array}{l}\text { Publication } \\
\text { year }\end{array}$ & Country & $\begin{array}{l}\text { Mean age/ } \\
\text { median }\end{array}$ & $\begin{array}{c}\text { Cases } \\
\text { (total } \\
\text { number) }\end{array}$ & Main findings \\
\hline Ahmad N, et al. & 2015 & Pakistan & $31.5+/-14.7$ & 181 & $\begin{array}{c}\text { Unsuccessful outcomes had a statistically significantly positive association } \\
\text { with age }>40 \text { years, baseline weight, } 40 \mathrm{~kg} \text {, concurrent comorbidities, resistance } \\
\text { to OFX, lung cavitation on baseline CXR, and treatment modification due to } \\
\text { adverse drug effects. }\end{array}$ \\
\hline Bastard M, et al. & 2015 & Armenia & 40 & 239 & $\begin{array}{l}\text { Reason for drug interruption include: patient refused treatment, adverse effects, } \\
\text { comorbid conditions, and severe conditions. }\end{array}$ \\
\hline \multirow[t]{2}{*}{ Mirsaeidi S, et al. } & 2005 & Iran & 34.1 & 17 & $\begin{array}{l}\text { TB treatment Iwas } 27.9 \text { (IQR 12-96) months; patients were, on average, resistant } \\
\text { to } 3.6 \text { drugs; }\end{array}$ \\
\hline & & & & & MDR-TB treatment was initiated with an average of 5.23 drugs. \\
\hline
\end{tabular}

95 distinct genotypes of Mycobacterium tuberculosis were identified, which belonged to three evolutionary groups (1-3). There were $86(90 \%)$ unique spoligotype patterns in 151 patients.

\begin{tabular}{lclccc}
\hline $\begin{array}{l}\text { Sanchez-Padilla E, } \\
\text { et al. }\end{array}$ & 2014 & Armenia & NR & 486 & $\begin{array}{c}\text { All patients interviewed mentioned difficulties in dealing with the side effects. } \\
\text { The second factor most frequently mentioned was fear that the treatment was not } \\
\text { effective or even harmful. }\end{array}$ \\
\hline $\begin{array}{l}\text { Tahaoğlu K, et al. } \\
\text { 2001 }\end{array}$ & Turkey & $15-68$ & 158 & $\begin{array}{c}\text { The percentage of patients with microbes that were resistant to more than five } \\
\text { drugs was significantly higher in the group with unsuccessful outcomes. }\end{array}$ \\
\hline
\end{tabular}

NR: not reported; OFX: ofloxacin; IQR: interquartile range; CXR: chest X-ray; MDR-TB: multidrug resistant tuberculosis.

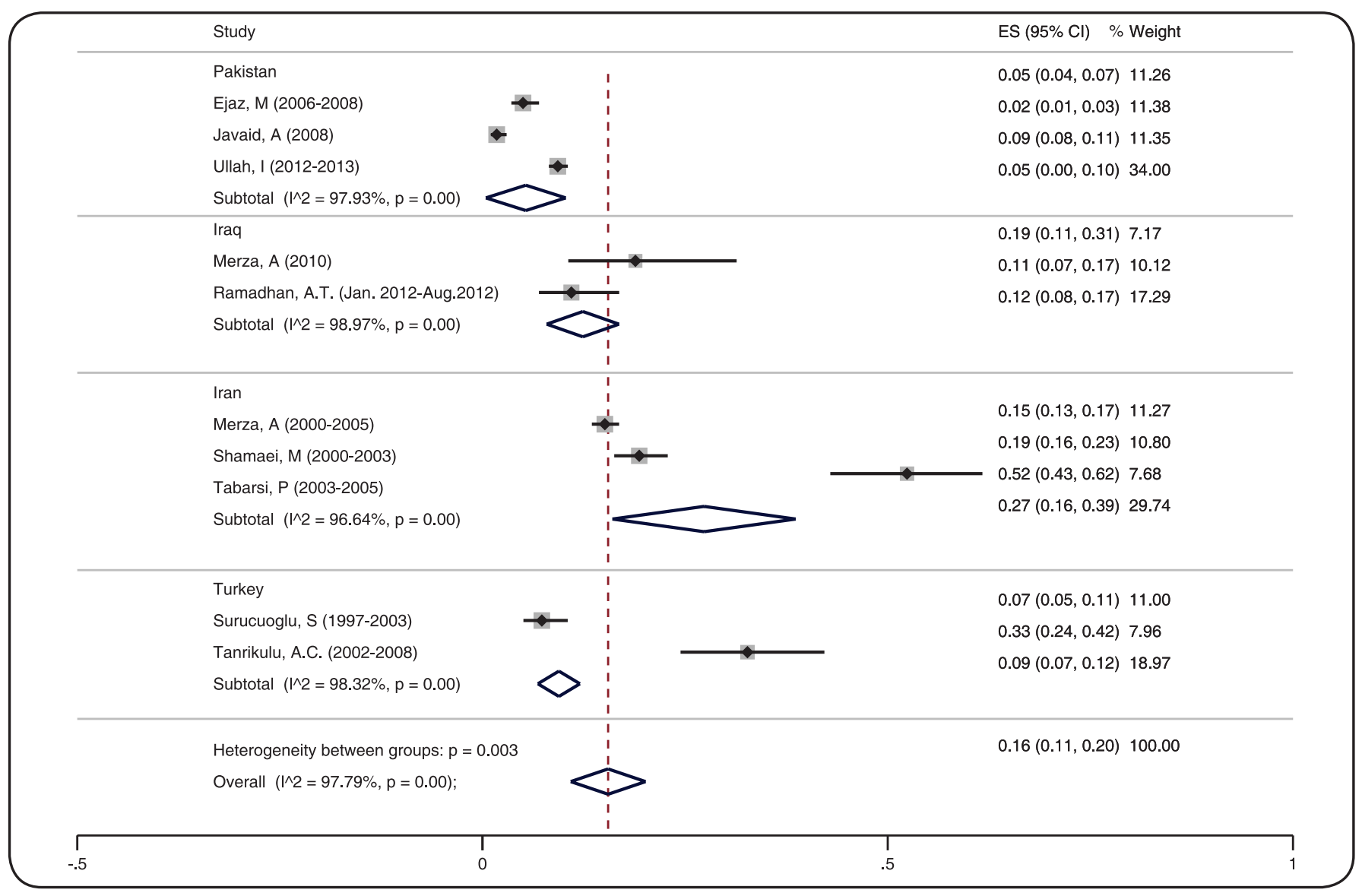

FIGURE 2 - Forest plot of the prevalence of multidrug resistant tuberculosis for the selected studies. ES: Estimates; CI: confidence interval. 


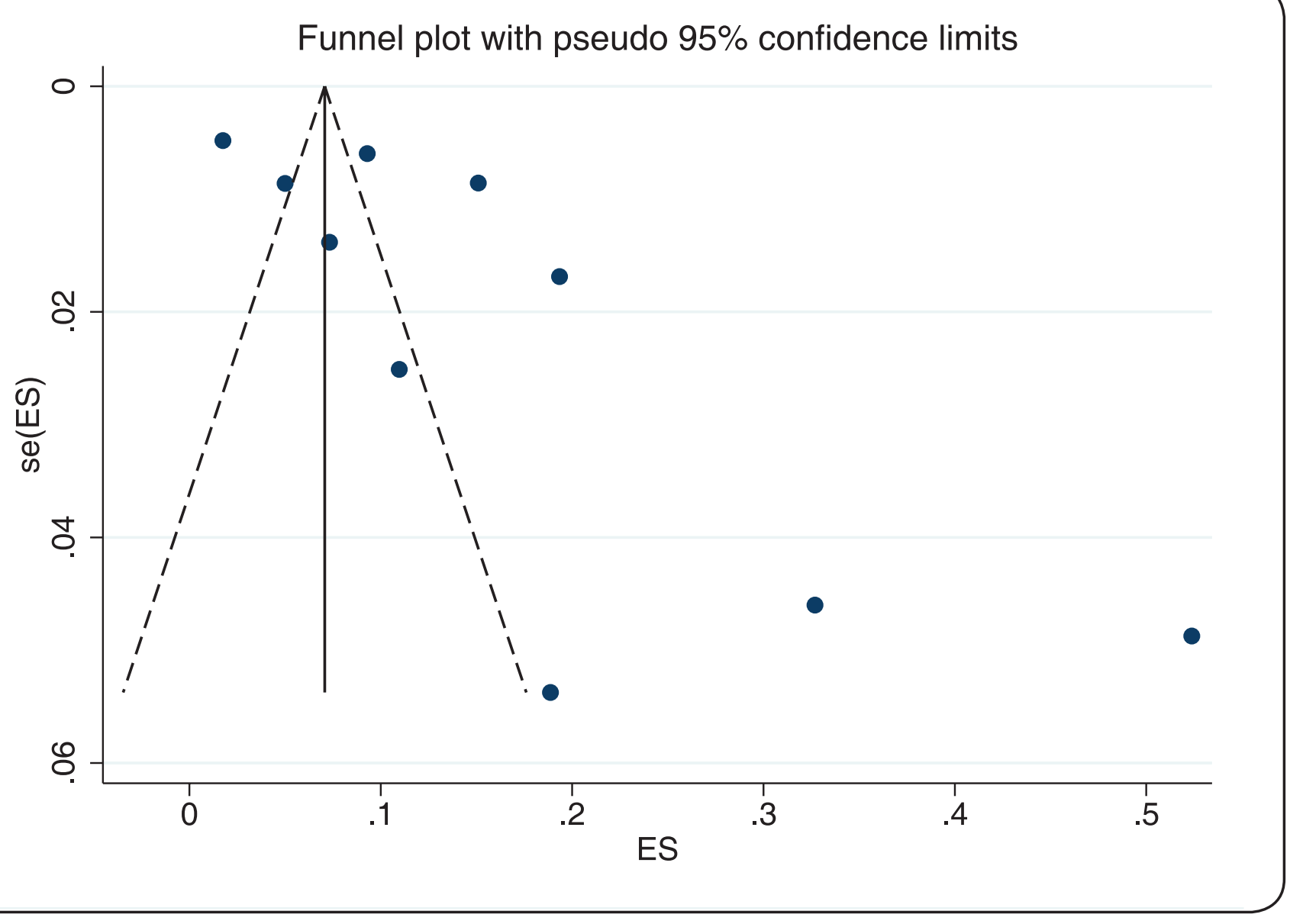

FIGURE 3 - Funnel plot for the meta-analysis of the prevalence of multidrug resistant tuberculosis in Iran and its neighboring countries. ES: estimates.

1.19-2.48) had an increased pool risk of developing MDR-TB. There was no heterogeneity between the studies for the same groups for all three risk factors, $\left(\mathrm{I}^{2}=0, \mathrm{P}<0.001\right)$. The forest plot revealed that the overall odds for the development of MDR-TB were 2.01 (95\% CI 1.65-2.36).

\section{DIscussion}

We found that the majority of studies included reported that many drugs were administered in the treatment of MDR-TB, most of which were accompanied by a multitude of adverse effects. Additionally, we found that prevalence rates ranged between 5-27\% in Pakistan, Turkey, Iraq, and Iran. The three risk factors identified with the development of MDR-TB were previous TB treatment history, those aged $<45$ years, and those who were males.

In this review, we found that MDR-TB cases in the countries investigated can be treated if diagnosed. However, the MDR-TB treatment duration ranges between 20-24 months, thus having implications for the cost of treatment and the long-term care, particularly retention in care, of those infected. A previous study conducted in Iran by Masjedi et al. ${ }^{2}$, reported that the costs of MDR-TB treatment are very high; MDR-TB is approximately 100-300 times more expensive than the treatment of drug sensitive TB (DS-TB). Another study conducted in the United Kingdom, indicated that the average cost to manage a pulmonary MDR-TB case exceeded 60,000 pounds, whereas for DS-TB it was only 6,040 pounds $^{32}$. This is an underestimation of the true costs, taking no account of the numerous factors that make managing such patients more expensive than an average hospitalized patient. The adverse effects of the drugs included in the MDR-TB regimen are very challenging for patients to deal with, sometimes forcing patients to discontinue their treatment. The studies by Ahmed et al. ${ }^{14}$ and Tahaoğlu et al..$^{28}$ reported a withdrawal or change of the MDR-TB treatment regimen as a result of the adverse effects.

The pooled estimate of the MDR-TB prevalence in Iran is higher than that in Iraq, Turkey, and Pakistan. Additionally, the prevalence in Iran $(27 \%, 95 \%$ CI 16-39) was higher than that of the overall point estimate for all of the studies included $(16 \%, 95 \%$ CI 11-20). The higher prevalence of MDR-TB in Iran when compared to the other countries in the region might be explained by the fact that Iran has a greater capacity to detect resistant bacilli. Iran is well resourced; it has more manpower and laboratory facilities available; thus, MDR-TB prevalence might actually be underestimated in the other countries. Moreover, the studies by Merza et al. ${ }^{21}$ and 


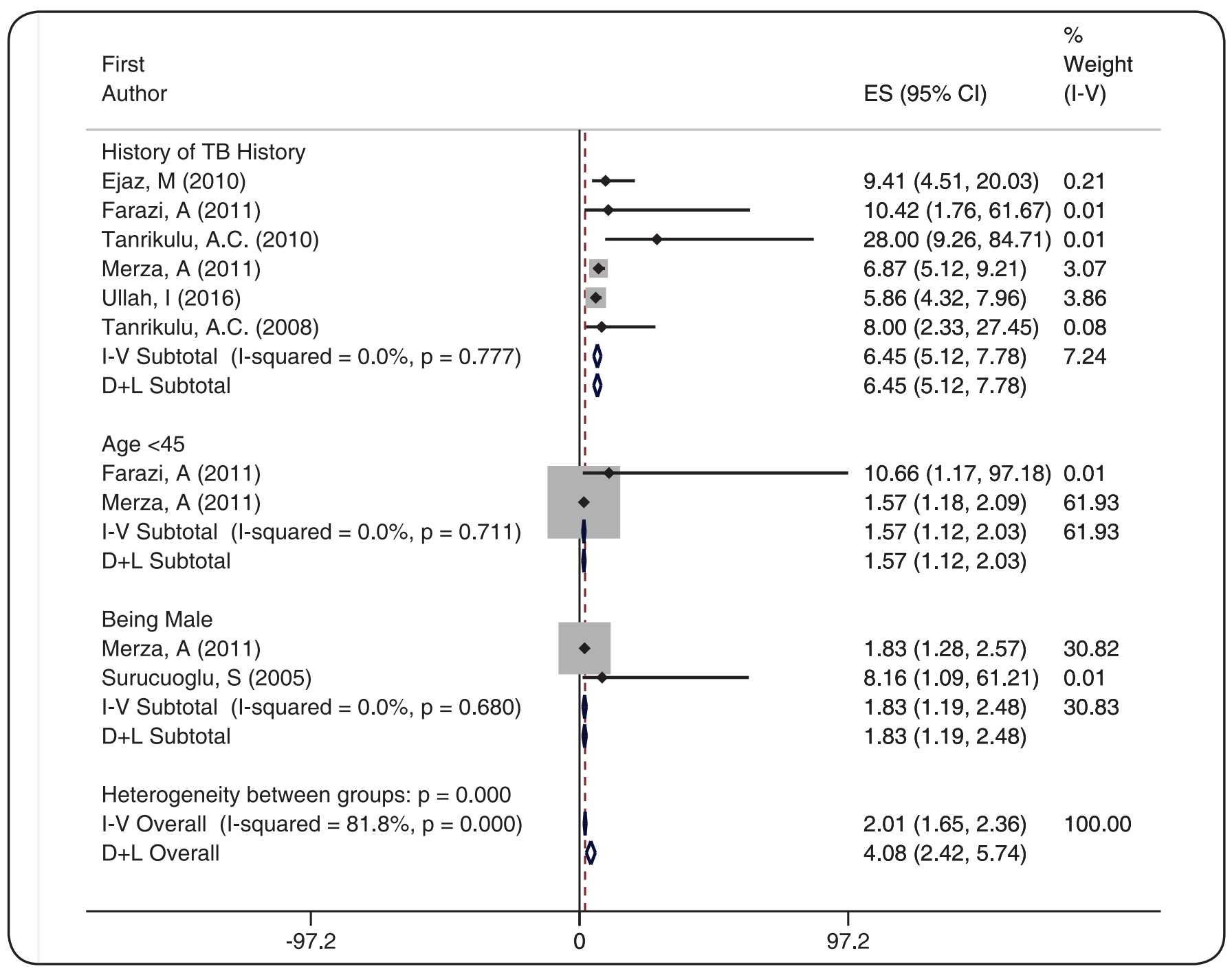

FIGURE 4 - Forest plot of the risk factors for development of multidrug resistant tuberculosis among the selected studies. ES: estimates; CI: confidence interval.

Shamaei et $a l .{ }^{25}$ which were conducted in Iran included $38.3 \%$ and 33.9\% migrant Afghan MDR-TB patients, respectively. A recent study by Hailie et $a l .{ }^{5}$ concluded that Iran was ranked as the world's largest refugee haven, predominately for Afghans, Iraqis, Pakistanis, Tajiks, Bosnians, and Azeris. It is possible that Central Asian Strain (CAS) family of TB, especially the SIT26 strain, might have been transported from Pakistan to the Sistan-Baluchestan province of Iran as this province shares geographic borders with Afghanistan and Pakistan where the CAS family is predominant. It has also been reported that due to the large numbers of immigrants living in Iran from Afghanistan, a country enlisted as one of the 22 high burden TB countries in the world, it is believed that there are considerable numbers of cases of active, latent, and multiple drug-resistant TB infection among Afghani refugees, and legal or illegal immigrants in Iran $^{6,7,33}$. Therefore, case detection efforts should be focused on migrant populations whose origin are from MDR-TB strain dominant countries, such as Afghanistan and Pakistan, in order to curb the continued MDR-TB transmission.
Previous TB treatment history, being aged less than 45 years, and being male were identified as the risk factors for MDR-TB among the included studies. These finding are in line with those from a similar study conducted in Europe by Faustini et al., who reported that the pooled odds of MDR-TB for those aged less than 45 years was 1.52 (95\% CI 1.13-2.03). In five of the eight studies they reviewed, the association between being aged less than 45 years and the risk of developing MDR-TB remained very high ${ }^{34}$. Additionally, Faustini et al. found that MDR-TB patients were more likely to be male, supporting the finding of the present systematic review and meta-analysis.

Another recent study conducted by $\mathrm{Merza}^{35}$ investigated hospitalized TB patients who were Afghani immigrants receiving tertiary care in Iran; risk factors for MDR-TB were being aged less than 45 years, being male, having a previous TB treatment history, poor socio-economic conditions, and smoking. This study revealed the dangers of the migration of people from MDR-TB hotspots to other regions or countries. This author highlighted the need for improving TB control 
programs inclusive of early detection of MDR-TB among Afghan immigrants, especially those with high risk factors for the development of MDR-TB.

This study was subject to some inherent limitations. For some of the countries considered for inclusion in this systematic review, there were no studies included because our search did not return any relevant studies. However, it is possible that there are relevant studies published in other languages that did not meet eligibility criteria for inclusion in this review. Males are possibly more susceptible MDR-TB than females due to the differing responses to the bacilli and/or to the drugs. However, this finding requires further investigation by specialists in the field to identify the underlining causes as to why males are more susceptible to MDR-TB than females.

To date, MDR-TB remains a pressing issue for public health programs in number of countries; if transmission continues the implications on human life will be catastrophic. Most of the studies we used for this systematic review and meta-analysis reported that a great numbers of drugs, accompanied by toxic adverse effects, are used for the treatment of MDR-TB. Patients' intolerance of the adverse effects associated with treatment, has led to lack of adherence to MDR-TB treatment regimens. The prevalence of MDR-TB was higher in Iran than in the other countries included in this review. Previous TB treatment history, being aged less than 45 years, and being male sex were risk factors associated with MDR-TB. It is imperative that prevention programs be strengthened and patients at high risk of MDR-TB should be targeted in order to provide the appropriate treatment and curb the on-going community transmission of MDR-TB.

\section{Acknowledgements}

The authors acknowledge the Students' Scientific Research Center of Tehran University of Medical Sciences for the funding it provided to conduct this study.

\section{Financial support}

This systematic review and meta-analysis was funded by the Students' Scientific Research Center, at the Tehran University of Medical Sciences, Tehran, Iran

\section{Conflict of interest}

The authors declare that have no conflicts of interest.

\section{REFERENCES}

1. World Health Organization (WHO). Global tuberculosis report, 2016. Available from: http://www.who.int/tb/publications/global_report/en/

2. Masjedi MR, Tabarsi P, Marjani M, Shiva PB, Nasehi M, Mehdi M. Management of MDR-TB: Review of Iran's Experience. Tanaffos. 2013;12(1):6-15

3. Zignol M, Hosseini MS, Wright A, Lambregts-van Weezenbeek C, Nunn $\mathrm{P}$, Watt CJ, et al. Global incidence of multidrug-resistant tuberculosis. J Infect Dis. 2006;194(4):479-85.

4. World Health Organization (WHO). Treatment of tuberculosis: guidelines. 4th ed. WHO; 2010. 147p.
5. Haeili M, Darban-Sarokhalil D, Fooladi AA, Javadpour S, Hashemi A, Siavoshi F, et al. Spoligotyping and drug resistance patterns of Mycobacterium tuberculosis isolates from five provinces of Iran. Microbiology Open. 2013;2:988-96.

6. Mirsaeidi SM, Tabarsi P, Khoshnood K, Pooramiri MV, RowhaniRahbar A, Mansoori SD, et al. Treatment of multiple drug-resistant tuberculosis (MDR-TB) in Iran. Int J Infect Dis. 2005;9(6):317-22.

7. Doroudchi M, Kremer K, Basiri EA, Kadivar MR, Van Soolingen D, Ghaderi AA. IS6110-RFLP and spoligotyping of Mycobacterium tuberculosis isolates in Iran. Scand J Infect Dis 2000;32(6):663-8.

8. Farnia P, Masjedi MR, Mirsaeidi M, Bahadori M, Velayati AA. Prevalence of Haarlem I and Beijing types. J Infect. 2006; 53:331-6.

9. Moher D, Liberati A, Tetzlaff J, Altman DG. Preferred reporting items for systematic reviews and meta-analyses: the PRISMA statement. Ann Intern Med. 2009;151(4):264-9.

10. Higgins J, Thompson SG. Quantifying heterogeneity in a meta-analysis. Statistics in medicine. Stat Med 2002;21:1539-58.

11. DerSimonian R, Laird N. Meta-analysis in clinical trials. Controlled clinical trials. Control Clin Trials. 1986;7:177-88.

12. Abdulahi A, Shab-Bidar S, Rezaei S, Djafarian K. Nutritional status of under five children in Ethiopia: a systematic review and meta-analysis. Ethiop J Health Sci. 2017;27(2):175-88.

13. Egger M, Davey-Smith G, Altman D. Systematic reviews in health care: meta-analysis in context. 2nd ed. London: BMJ Publishing; 2001. 506p.

14. Ahmad N, Javaid A, Basit A, Afridi AK, Khan MA, Ahmad I, et al. Management and treatment outcomes of MDR-TB: results from a setting with high rates of drug resistance. Int $J$ Tuberc Lung Dis. 2015;19(9):1109-14

15. Bastard M, Sanchez-Padilla E, Hewison C, Hayrapetyan A, Khurkhumal $\mathrm{S}$, Varaine $\mathrm{F}$, et al. Effects of treatment interruption patterns on treatment success among patients with multidrug-resistant tuberculosis in Armenia and Abkhazia. J Infect Dis. 2015; 211(10):1607-15.

16. Ejaz M, Siddiqui AR, Rafiq Y, Malik F, Channa A, Mangi R, et al. Prevalence of multi- drug resistant tuberculosis in Karachi, Pakistan: identification of at risk groups. Trans R Soc Trop Med Hyg. 2010;104(8):511-7.

17. Farazi A, Jabbariasl M, Sofian M. Drug resistance among tuberculosis patients in central province-Iran (2005-2010). Trop Med Int Health 2011;16:373.

18. Javaid A, Hasan R, Zafar A, Ghafoor A, Pathan AJ, Rab A, et al. Prevalence of primary multidrug resistance to anti-tuberculosis drugs in Pakistan. Int J Tuberc Lung Dis. 2008;12(3):326-31.

19. Masjedi MR, Tabarsi P, Chitsaz E, Baghaei P, Mirsaeidi M, Amiri MV, et al. Outcome of treatment of MDR-TB patients with standardised regimens, Iran, 2002-2006. Int J Tuberc Lung Dis. 2008;12(7):750-5.

20. Merza MA, Farnia P, Masjedi MR, Ridell M. Anti-tuberculosis drug resistance in Dohuk, Iraq. Int J Tuberc Lung Dis. 2010;14(9):1213-4.

21. Merza MA, Farnia P, Tabarsi P, Khazampour M, Masjedi MR, Velayati AA. Anti- tuberculosis drug resistance and associated risk factors in a tertiary level TB center in Iran: a retrospective analysis. J Infect Dev Ctries. 2011;5(7):511-9.

22. Ramadhan AT, Mankhi AM. The pattern of drug resistance in Iraqi pulmonary tuberculosis patients today, MDR-TB is a big concern for public health issue in number of countries and if the trend continues at is human life catastrophe is unavoidable referred to the specialized center for chest and respiratory disease. Iraqi Postgrad Med J. 2014;13(2):181-6.

23. Ramazanzadeh R, Farnia P, Amirmozafari N, Ghazi F, Ghadertotonchi Z, Kamran J, et al. Comparison between molecular epidemiology, geographical regions and drug resistance in Mycobacterium tuberculosis strains isolated from Iranian and Afghan patients. Chemotherapy. 2006;52(6):316-20.

24. Sanchez-Padilla E, Marquer C, Kalon S, Qayyum S, Hayrapetyan A, Varaine $\mathrm{F}$, et al. Reasons for defaulting from drug-resistant tuberculosis 
treatment in Armenia: a quantitative and qualitative study. The international journal of tuberculosis and lung disease: Int J Tuberc Lung Dis. 2014;18(2):160-7.

25. Shamaei M, Marjani M, Chitsaz E, Kazempour M, Esmaeili M, Farnia $\mathrm{P}$, et al. First- line anti-tuberculosis drug resistance patterns and trends at the national TB referral center in Iran-eight years of surveillance. Int J Infect Dis. 2009;13(5):236-40.

26. Surucuoglu S, Ozkutuk N, Celik P, Gazi H, Dinc G, Kurutepe S, et al. Drug-resistant pulmonary tuberculosis in western Turkey: prevalence, clinical characteristics and treatment outcome. Ann Saudi Med. 2005;25(4):313-8.

27. Tabarsi P, Nooraki A, Mirsaeidi M, Amiri M, Baghaei P, Farnia P, et al. Representative drug susceptibility patterns for guiding design of re-treatment regimens for multidrug-resistant tuberculosis in Iran. Respirology. 2008;13(1):108-11.

28. Tahaoğlu K, Törün T, Sevim T, Ataç G, Kir A, Karasulu L, et al. The treatment of multidrug-resistant tuberculosis in Turkey. N Engl J Med 2001;345(3):170-4.

29. Tanrikulu AC, Abakay A, Abakay O. Risk factors for multidrugresistant tuberculosis in Diyarbakir, Turkey. Medical science monitor: Med Sci Monit. 2010;16(6):PH57-62.
30. Tanrikulu AC, Hosoglu S, Ozekinci T, Abakay A, Gurkan F. Risk factors for drug resistant tuberculosis in southeast Turkey. Trop Doct. 2008;38(2):91-3.

31. Ullah I, Javaid A, Tahir Z, Ullah O, Shah AA, Hasan F, et al. Pattern of drug resistance and risk factors associated with development of drug resistant Mycobacterium tuberculosis in Pakistan. PloS One. 2016;11(1):e0147529.

32. White V, Moore-Gillon J. Resource implications of patients with multidrug resistant tuberculosis. Thorax. 2000;55:962-3.

33. Feyisa SG, Haeili M, Zahednamazi F, Mosavari N, Taheri MM, Hamzehloo G, et al. Molecular characterization of Mycobacterium tuberculosis isolates from Tehran, Iran by restriction fragment length polymorphism analysis and spoligotyping. Rev Soc Bras Med Trop. 2016;49:204-10.

34. Faustini A, Hall AJ, Perucci CA. Risk factors for multidrug resistant tuberculosis in Europe: a systematic review. Thorax. 2006;61(2):15863.

35. Merza MA. Risk factors for multi-drug resistant tuberculosis of afghan immigrants in a tertiary care hospitalized tuberculosis patients in Iran. IJCR 2015:7(1):12032-12035. 